\title{
Ferromagnetic transition of a two-component Fermi gas of hard spheres
}

\author{
F. Arias de Saavedra \\ Departamento de Física Atómica, Molecular y Nuclear, Universidad de Granada, 18071 Granada, Spain \\ F. Mazzanti and J. Boronat \\ Departament de Física i Enginyeria Nuclear, and Campus Nord B4-B5, Universitat Politècnica de Catalunya, \\ 08034 Barcelona, Spain
}

\author{
A. Polls \\ Departament d'Estructura i Constituents de la Matèria and Institut de Ciències del Cosmos, \\ and Universitat de Barcelona, 08028 Barcelona, Spain \\ (Received 28 October 2011; published 12 March 2012)
}

\begin{abstract}
We use microscopic many-body theory to analyze the problem of itinerant ferromagnetism in a repulsive atomic Fermi gas of hard spheres. Using simple arguments we show that the available theoretical predictions for the onset of the ferromagnetic transition predict a transition point at a density $\left(k_{F} a \sim 1\right)$ that is too large to be compatible with the universal low-density expansion of the energy. We present variational calculations for the hard-sphere Fermi gas, in the framework of Fermi hypernetted chain theory, that shift the transition to higher densities $\left(k_{F} a \sim 1.8\right)$. Backflow correlations, which are mainly active in the unpolarized system, are essential for this shift.
\end{abstract}

DOI: 10.1103/PhysRevA.85.033615

PACS number(s): 67.85.-d, 05.30.Fk, 75.25.-j

\section{INTRODUCTION}

Experimental and theoretical progress over the last decade has allowed for a deeper understanding of phenomena such as superfluidity and pairing along the BEC-BCS crossover in degenerate Fermi gases [1]. In these studies, the diluteness condition of the system allows for a characterization of the interaction by its $s$-wave scattering length $a$, a quantity that is easily tuned by making the system approach a Feshbach resonance. In most cases, however, the underlying interaction between atoms is short ranged and attractive, while little attention has been paid to the case of short-range repulsive potentials.

The role played by short-range repulsive interactions has been revived more recently with a series of experiments discussing the possible onset of a phase transition to a ferromagnetic state in two-component ultracold Fermi gases. In an initial work, Jo et al. [2] describe an experiment with ${ }^{6} \mathrm{Li}$ atoms which is supposed to implement the Stoner model $[3,4]$ of magnetic interactions, and find clear signatures of the ferromagnetic transition by monitoring the total and kinetic energy of the gas and its volume. They conclude from these measurements that a transition to the ferromagnetic state occurs around $x=k_{F} a \approx 1.9 \pm 0.2$ with $k_{F}$ the Fermi momentum of the unpolarized system. In a second and more recent experiment [5], the existence of a ferromagnetic transition in the same system is ruled out, arguing that very fast molecule formation leads to local heating and to a pairing instability, destroying the ultracold nature of the gas. They even conclude that short-range repulsive interactions with fermionic species can possibly not be realized in nature.

The existence of a ferromagnetic phase in dilute, ultracold Fermi gases interacting through short-range repulsive forces has also been discussed in recent theoretical works, triggered by the previous experiments [2,5]. Positive scattering lengths can be achieved by moving along the upper branch of a
Feshbach resonance associated to an attractive short-range interaction once a bound state is formed, or by increasing the range of an overall repulsive potential. These two mechanisms lead to different physical situations, as pointed out in Refs. [6,7], when a description based only on the scattering length is not enough. However, in both cases it can be argued that when the system is so dilute that the scattering length and the range of the potential are small compared to the interparticle distance, only $s$-wave scattering processes are relevant. Under these conditions, all higher order partial waves can be discarded in the description of the many-body system. In any case and motivated by the previous experiments, the question of whether a ferromagnetic transition takes place in ultracold Fermi gases has achieved revived interest recently. However, no consensus has been reached yet. For instance, in Refs. [8-11] the authors find that a transition exists in different systems of harmonically trapped repulsive fermions, while similar conclusions regarding infinite systems are reported in Refs. $[6,12,13]$. In other works, the existence of a transition is either ruled out or argued to depend on the details of the system analyzed $[14,15]$. In this work we address this problem again and analyze the possible onset of a ferromagnetic transition in a system of fermions interacting through purely repulsive, short-ranged forces. We believe that the question of weather a system of repulsive fermions can undergo a ferromagnetic transition is still relevant even though in actual experiments the high $x$ limit is usually reached by moving along the upper branch of a Feshbach resonance.

\section{MODELS}

In the case of a fully polarized Fermi system, $s$-wave scattering is forbidden by the Pauli principle, and thus the total energy equals the corresponding kinetic energy of the underlying Fermi sea if the density is low enough. In a nonpolarized medium, $s$-wave scattering between particles 
with the same spin orientation is also suppressed but not between atoms of different spin. All that information can be collected in the following, effective opposite-spin-channel (OSC) Hamiltonian

$$
H_{\mathrm{OSC}}=-\frac{\hbar^{2}}{2 m}\left(\sum_{i=1}^{N_{\uparrow}} \nabla_{i}^{2}+\sum_{i^{\prime}=1}^{N_{\downarrow}} \nabla_{i^{\prime}}^{2}\right)+\sum_{i, i^{\prime}}^{N_{\uparrow}, N_{\downarrow}} V\left(r_{i i^{\prime}}\right),
$$

with $m$ the mass of each of the $N=N_{\uparrow}+N_{\downarrow}$ atoms, $N_{\uparrow}$ and $N_{\downarrow}$ being the number of particles with spin up and spin down, respectively. The Stoner model [3,4] corresponds to a mean-field approach to this Hamiltonian when the interaction is replaced by a two-body pseudopotential with a coupling constant proportional to the $s$-wave scattering length $a$. As commented above, this model has been taken as the starting point in recent theoretical analysis of the ferromagnetic transition. Contact interactions in standard Monte Carlo simulations of dilute quantum gases are usually replaced by model potentials of finite range tuned to reproduce the desired values of scattering parameters. One of the fully repulsive potential models commonly used to study low-density properties of degenerate quantum gases is the hard-sphere interaction,

$$
V(r)= \begin{cases}+\infty & r \leqslant R \\ 0 & \text { otherwise }\end{cases}
$$

with an $s$-wave scattering length $a$ equal to the core diameter $R$, which been used recently in Refs. $[6,13,16]$.

When a finite range potential is explored, higher order partial waves produce contributions that show up when the diluteness condition of the gas is not fulfilled. In this case the OSC model, which explicitly restricts this possibility, shows its limitations. When applied to the experiment with ${ }^{6} \mathrm{Li}$ atoms of Ref. [2], all models predict a ferromagnetic transition at a point where the system is clearly not dilute. With $k_{F}$ the Fermi momentum of the paramagnetic phase, the Stoner model predicts the transition at $k_{F} a=\pi / 2$, while the Monte Carlo simulations of Refs. $[6,13]$ lower that prediction to $k_{F} a \approx 0.9$, with minor differences between the case of a fully repulsive interaction and a short-ranged attractive one. With a transition point at $k_{F} a \sim 1$, it is no longer evident that the OSC model can still be used to describe the system. In this work we study the possible onset of a ferromagnetic transition in a spin $1 / 2$ Fermi gas of hard spheres (i.e., a two-component Fermi gas) using existing low-density expansions of the energy per particle and a variational approach in the framework of the Fermi hypernetted chain equations (FHNC) [18,19]. As we do not restrict the analysis to the weakly interacting regime, we allow for the contribution of scattering in all partial waves by adopting a more general Hamiltonian of the form

$$
\begin{aligned}
H= & -\frac{\hbar^{2}}{2 m}\left(\sum_{i=1}^{N_{\uparrow}} \nabla_{i}^{2}+\sum_{i^{\prime}=1}^{N_{\downarrow}} \nabla_{i^{\prime}}^{2}\right) \\
& +\sum_{i<j}^{N_{\uparrow}} V\left(r_{i j}\right)+\sum_{i^{\prime}<j^{\prime}}^{N_{\downarrow}} V\left(r_{i^{\prime} j^{\prime}}\right)+\sum_{i, i^{\prime}}^{N_{\uparrow}, N_{\downarrow}} V\left(r_{i i^{\prime}}\right),
\end{aligned}
$$

where indexes $i, j, \ldots$ and $i^{\prime}, j^{\prime}, \ldots$ label spin-up and spindown particles, respectively. We stick to the case where the two-body interaction does not distinguish between different spin configurations, but explicitly include interactions between spins with the same orientation, in contrast to the OSC model. Since we are analyzing the gas of hard-sphere fermions, we use (2) for $V(r)$ in all three channels (up-up, down-down, and up-down).

\section{RESULTS}

We are mainly interested in the comparison between the energy per particle of the paramagnetic, unpolarized system $\left(N_{\uparrow}=N_{\downarrow}=N / 2\right)$ and the ferromagnetic, fully polarized one $\left(N_{\uparrow}=N\right)$. The density determines the Fermi momentum, which is different in each case due to the spin degeneracy. For the polarized and unpolarized systems one has $k_{F}^{\mathrm{P}}=\left(6 \pi^{2} \rho\right)^{1 / 3}$ and $k_{F}^{\mathrm{NP}}=\left(3 \pi^{2} \rho\right)^{1 / 3}$, respectively, so $k_{F}^{\mathrm{P}}=2^{1 / 3} k_{F}^{\mathrm{NP}}$. As in previous works, we set $k_{F}=k_{F}^{\mathrm{NP}}$ as the momentum scale unit and plot our results in terms of the dimensionless quantity $x=k_{F} a$. Due to the spin degeneracy, the paramagnetic phase is preferred in the noninteracting $a \rightarrow 0$ limit at zero temperature. The stability of the system when the polarization $\Delta=\left(N_{\uparrow}-N_{\downarrow}\right) / N$ increases is determined by the inverse magnetic susceptibility $\chi^{-1}$,

$$
\frac{1}{\chi}=\frac{1}{\rho}\left(\frac{\partial^{2} E / N}{\partial \Delta^{2}}\right)_{\Delta=0} .
$$

The critical density against spin fluctuations corresponds to the point where $\chi^{-1}\left(x_{c}\right)=0$. On the other hand, a sufficient condition indicating that the ferromagnetic phase is preferred is given by the criteria $E^{\mathrm{P}}\left(x_{c}^{\prime}\right)-E^{\mathrm{NP}}\left(x_{c}^{\prime}\right)=0$, with $E$ the energy of the system. This second estimation, which is the one we use in the present work, is fulfilled at a slightly larger density than the first one $\left(x_{c} \leqslant x_{c}^{\prime}\right)$. For instance, in the Stoner model $x_{c}=\pi / 2 \simeq 1.57$ while $x_{c}^{\prime}=(9 \pi / 10)\left(2^{2 / 3}-1\right) \simeq 1.66$.

Several expressions for the ground state energy per particle of a system of interacting fermions at low densities have been derived in the past [20-22]. All of them rely on perturbation theory in terms of a renormalized, effective interaction obtained from a $G$ matrix which contains information about the multiple scattering of particles moving on the correlated medium. For a strong repulsive potential the massive summation of selected terms is a convenient strategy to get a rapidly convergent perturbation series, and becomes absolutely mandatory for the extreme case of the hard-sphere potential due to the divergent character of the matrix elements of the bare potential. A very useful expansion containing terms beyond $s$-wave contributions is the one derived by Bishop in Ref. [22], which is expressed in terms of the $s$ - and $p$-wave scattering lengths $a$ and $a_{p}$, respectively, and $r_{0}$, the $s$-wave effective range, as

$$
\begin{aligned}
\frac{E}{N}= & \frac{\hbar^{2} k_{F}^{2}}{2 m}\left[\frac{3}{5}+(v-1)\left(\frac{2}{3 \pi}\left(k_{F} a\right)+\frac{4(11-2 \ln 2)}{35 \pi^{2}}\left(k_{F} a\right)^{2}\right.\right. \\
& \left.+\frac{1}{10 \pi}\left(k_{F} r_{0}\right)\left(k_{F} a\right)^{2}+[0.076+0.057(v-3)]\left(k_{F} a\right)^{3}\right) \\
& +\frac{1}{5 \pi}(v+1)\left(k_{F} a_{p}\right)^{3}+(v-1)(v-2) \frac{16}{27 \pi^{3}}\left(4 \pi-3^{3 / 2}\right) \\
& \left.\times\left(k_{F} a\right)^{4} \ln \left(k_{F} a\right)+\cdots\right]
\end{aligned}
$$


with $v=1(2)$ the spin degeneracy of the polarized (unpolarized) system. In this expansion, the $p$-wave scattering length is defined in terms of the $p$-wave phase shift $\delta_{1}(k)$ as $a_{p}=\left.\left[-k^{3} \cot \delta_{1}(k) / 3\right]^{-1 / 3}\right|_{k \rightarrow 0}$. For the particular case of the hard-sphere interaction, where $a_{p}=a$ and $r_{0}=2 a / 3$, the previous expression reduces to the one originally derived by Efimov [20].

For an unpolarized system of hard spheres and with $x=$ $k_{F} a$, the previous expression reduces to

$$
\frac{E^{\mathrm{NP}}}{N}=\frac{\hbar^{2} k_{F}^{2}}{2 m}\left[\frac{3}{5}+\frac{2}{3 \pi} x+\frac{4(11-2 \ln 2)}{35 \pi^{2}} x^{2}+0.231 x^{3}\right],
$$

while for the polarized system one recovers

$$
\frac{E^{\mathrm{P}}}{N}=\frac{\hbar^{2} k_{F}^{2}}{2 m}\left[\frac{3}{5}+\frac{2}{5 \pi} x^{3}\right] .
$$

Notice that in these expressions and up to order $x^{3}, E^{\mathrm{NP}} / N$ contains $s$ - and $p$-wave contributions besides the kinetic energy of the corresponding free Fermi sea, while $s$-wave effects are suppressed in $E^{\mathrm{P}} / N$. However, there is still a $p$-wave contribution of order $x^{3}$ that becomes important when the density increases. This can be seen in Fig. 1, where we compare the FN-DMC results of Refs. [13] (black circles) and [6] (red squares) for the unpolarized gas to different instances of Eq. (6) obtained by keeping all terms (black solid line), removing the $p$-wave contributions (violet dotted line), or keeping only the universal terms (green dot-dashed line), which are of order $x^{2}$ and depend on the interaction only through the $s$-wave scattering length

$$
\frac{E_{u}^{\mathrm{NP}}}{N}=\frac{\hbar^{2} k_{F}^{2}}{2 m}\left[\frac{3}{5}+\frac{2}{3 \pi} x+\frac{4}{35 \pi^{2}}(11-2 \ln 2) x^{2}\right] .
$$

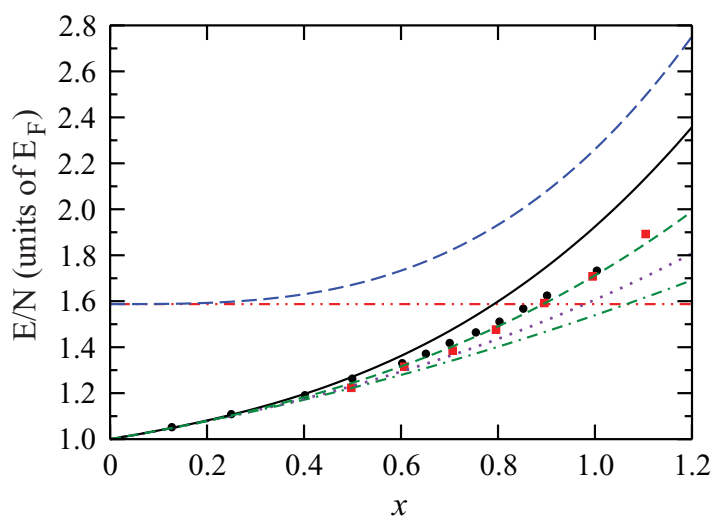

FIG. 1. (Color online) Energy per particle of the polarized and unpolarized hard-sphere Fermi gases. The black solid, violet dotted, and green dot-dashed lines show the low-density expansion of Eq. (5) for the unpolarized system, the same with the $p$-wave contribution (proportional to $a_{p}^{3}$ ) removed, and the universal prediction of Eq. (8). The blue dashed line depicts the prediction for the polarized system given in Eq. (7). The red dot-dot-dashed straight line is the constant value assigned to the polarized system in the OSC model, while the red squares and solid black circles stand for the DMC calculations of Refs. [6,13] for the unpolarized system in the same approximation.
To this same order, $E^{\mathrm{P}} / N$ reduces to the kinetic energy of the underlaying polarized Fermi sea (red dot-dot-dashed line).

The previous expansions are asymptotic in nature and so it is difficult to accurately determine its convergence radius. However, in the particular case of a hard-sphere interaction, they are known to reproduce the equation of state up to relatively large values of $x$. Furthermore, they cannot be directly compared to the OSC model as it is implicitly assumed in their derivation that the Hamiltonian contains the same interatomic potential in all channels. It is however possible to obtain a prediction for the OSC model if the leading terms in the expansion are renormalized accordingly. The OSC model discards interactions between particles with the same spin orientation, which in a first approximation can be thought as forming pairs in a triplet state of total spin $S=1$. The wave function in configuration space for these pairs is therefore totally antisymmetric and thus $s$-wave scattering processes between them are suppressed. In this way and to leading order, the $s$-wave contributions in Eq. (5) should be the same in the two model Hamiltonians of Eqs. (1) and (3). For $S=1$ states, $p$-wave scattering is the leading contribution, but only the symmetric combination of unparallel spins is allowed in the OSC model. Assuming all three spin configurations contribute the same to the triplet state in the Hamiltonian $H$ in Eq. (3), we conclude that the $p$-wave contribution to $H_{\mathrm{OSC}}$ should be one third of the $p$-wave contribution to $H$. Taking all these facts into account, we can build an expansion of the energy per particle of the OSC model by simply weighting with an extra factor $1 / 3$ the term proportional to $a_{p}^{3}$ in Eq. (5). Doing so leads to the green dashed line in Fig. 1, which accurately reproduces the Monte Carlo calculations of Refs. [6,13] which were obtained from the model Hamiltonian $H_{\mathrm{OSC}}$. Accordingly, one may conclude that the low-density expansions given above are valid in the whole range of $x$ values considered, and that both $s$ - and $p$-wave scattering processes contribute significantly to the total energy per particle when $x$ increases.

Assuming then that the above low-density expansions provide a good description of the energy per particle of the polarized and unpolarized systems at $x \sim 1$, one can draw several conclusions from the different curves in Fig. 1. For instance, one sees from the figure that the $x^{3}$ terms add important contributions to $E_{u}^{\mathrm{NP}} / N$. Adding only $x^{3}$ terms to the $s$ wave raises the energy per particle slightly, but the most significant effect appears when the $p$-wave contributions are added, bringing the violet dotted line into the black solid line corresponding to the prediction for the full model of Eq. (3). Therefore, adding $p$-wave effects is more important than dressing the $s$-wave contributions by including the effective range terms. Furthermore, the good agreement between the Monte Carlo predictions and the properly modified lowdensity expansion for the OSC model, indicates that while $p$-wave contributions are important, no higher order partial waves contribute significantly, as these are not included in the expansion. We thus conclude that $s$ - and $p$-wave processes are the only ones that contribute significantly.

It is also apparent from Fig. 1 that the universal behavior, where all predictions yield essentially the same, ceases to be valid already at $x_{0} \sim 0.4$, quite below the value $x \sim 1$ where the simulations based on the OSC model predict the transition to the ferromagnetic phase. The figure also indicates that the 
energy per particle of the polarized gas starts to deviate from the constant prediction, valid when only $s$-wave scattering is considered, at about the same value $x=x_{0}$. Once again, at $x \sim 1 p$-wave contributions introduce significant corrections that cannot be neglected. We thus conclude that, at least for the hard-sphere Fermi gas, the low-density expansions set the limit of validity of the OSC model at $x \sim x_{0}$.

Knowing the limits of the OSC model, the next step in our discussion is to analyze the results provided by the full Hamiltonian $H$ of Eq. (3) with the interaction acting in all the spin channels. To this end we perform a variational calculation in the framework of the Fermi hypernetted chain equations (FHNC) [18,19,23]. Although not exact, this method has been successfully used in the past to describe strongly interacting liquids like pure ${ }^{3} \mathrm{He}$ [24-26], ${ }^{3} \mathrm{He}-{ }^{4} \mathrm{He}$ mixtures [27], and nuclear matter [28,29], and is therefore expected to accurately describe the physics of the present problem, We use a variational Slater-Jastrow wave function of the form

$$
\Psi_{B}=\left[\prod_{i<j} f\left(r_{i j}\right)\right] D_{\uparrow}\left(\vec{r}_{1}, \vec{r}_{2}, \ldots, \vec{r}_{N_{\uparrow}}\right) D_{\downarrow}\left(\vec{r}_{1}^{\prime}, \vec{r}_{2}^{\prime}, \ldots, \vec{r}_{N_{\downarrow}}\right),
$$

where $f(r)$ is a two-body correlation factor, while $D_{\uparrow}$ and $D_{\downarrow}$ are Slater determinants of spin-up and spin-down plane waves filling momentum states up to the Fermi level, and including backflow correlations [30]. In our FHNC calculations, the sum of elementary diagrams is approximated using the interpolating equation approximation [24,25]. The results obtained with this wave function (9) are compared with those obtained from a less sophisticated version $\Psi$ where backflow correlations are removed. In both cases we take the Jastrow factor $f(r)$ to be the same for all spin channels, and equal to the solution of the optimal Euler-Lagrange hypernetted chain equations (HNC/EL) $[23,31]$ for the underlying Bose gas of atoms of the same mass $m$ interacting through the same potential and at the same density. We have checked that by using the optimal $f(r)$, the energy per particle improves noticeably compared with other, simpler forms containing few variational parameters, specially in the case of the unpolarized gas. On the other hand, backflow correlations enter exponentially in the Slater determinants of plane waves through the renormalized position coordinates [26]

$$
\mathbf{r}_{i} \rightarrow \tilde{\mathbf{r}}_{i}+\lambda \sum_{j \neq i}^{N} \eta\left(r_{i j}\right) \mathbf{r}_{i j}
$$

where

$$
\eta(r)=\exp \left[-\left(\frac{r-r_{0}}{\omega_{0}}\right)^{2}\right]
$$

with $\lambda, r_{0}$, and $\omega_{0}$ variational parameters that are optimized at each density. Backflow correlations are capital in an accurate description of the energetics of strongly correlated systems like ${ }^{3} \mathrm{He}[32,33]$, and have been reported to have also a nonnegligible impact in the problem considered here at the densities where the ferromagnetic transition is predicted [6].

Figure 2 shows our results for the total energy per particle of the polarized and unpolarized systems in different approx-

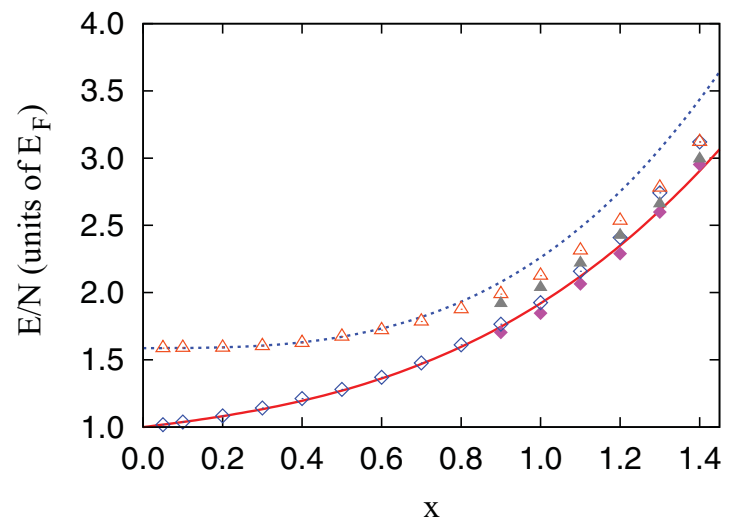

FIG. 2. (Color online) Energy per particle of the paramagnetic and ferromagnetic phases in different approximations. Solid and open diamonds: FHNC results for the unpolarized gas including or not backflow correlations, respectively. Solid and open triangles: same for the polarized system. The solid and dotted lines are the predictions of the expansions in Eqs. (6) and (7), respectively.

imations. The solid and dotted lines show the low-density expansions of Eqs. (6) and (7), respectively. The open triangles and diamonds correspond to the predictions for the polarized and unpolarized systems using the HNC/EL-optimized wave function of Eq. (9) without backflow correlations, while the solid symbols stand for the same predictions including backflow. Several conclusions can be drawn from this figure. On the one hand, it is remarkable the fact that while the lowdensity expansion for the unpolarized system is everywhere close to the full many-body calculations, this is not the case for the polarized system, which overestimates the total energy per particle of the gas when $x \geqslant 0.6$. On the other, while the low-density expansion predictions do not cross in the range of densities analyzed, our many-body calculations show that the energy per particle of the polarized system decreases at a higher rate than the corresponding one for the unpolarized gas. It is also remarkable to notice that the total energy per particle of both systems noticeably depends on the quality of the wave function employed. As expected, the one including backflow correlations provides lower variational estimations. In any case, the inclusion of backflow correlations not only lowers the energies but also pushes the ferromagnetic transition to a higher density, identified by the point in the figure where the energy of the paramagnetic state equals that of the ferromagnetic phase (a sufficient condition that determines a point $x_{c}^{\prime}$ where the ferromagnetic phase is preferred). The open symbols show that $\Psi$ predicts a transition at $x_{c}^{\prime} \sim 1.4$, while the solid symbols, corresponding to $\Psi_{B}$, approach each other but do not cross yet in the range of densities considered in the figure. In any case, it is relevant to realize that the use of a Hamiltonian allowing the interaction in all three spin channels (up-up, down-down, and up-down), together with a rich variational wave function, bring the transition point back from $x_{c}^{\prime} \sim 1$ to higher values closer to the prediction of the Stoner model, $x_{c}^{\prime} \sim 1.66$.

The precise density at which our fully correlated model predicts the transition to the ferromagnetic state can be determined by extending the range of $x$ values considered. In Fig. 3 we show our predictions for $E^{\mathrm{P}} / N$ and $E^{\mathrm{NP}} / N$ obtained 


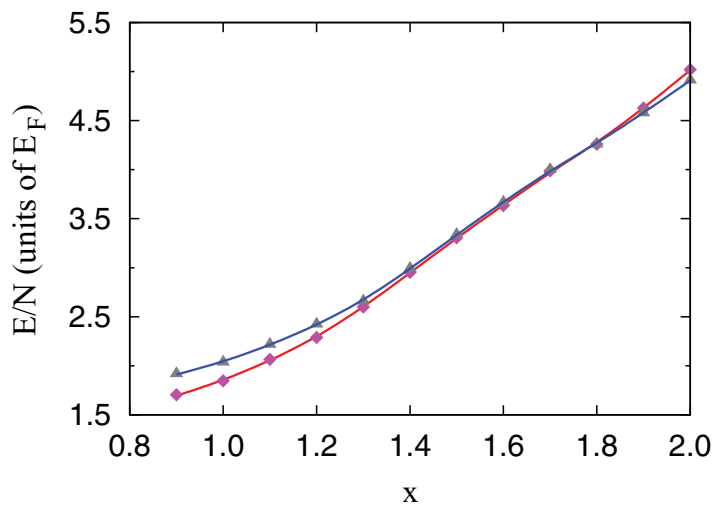

FIG. 3. (Color online) Energy per particle of the polarized (gray triangles) and unpolarized (red diamonds) system obtained from the HNC/EL-optimized wave function of Eq. (9) including backflow correlations, in the framework of the FHNC theory. The solid and dotted lines are a guide to the eye.

from the optimized wave function $\Psi_{B}$ of Eq. (9) including backflow correlations. As it can be seen, there is a wide range of densities where $E^{\mathrm{P}} / N$ and $E^{\mathrm{NP}} / N$ are fairly close to each other, and thus establishing a clear prediction of the transition point is delicate. Still, we clearly see that for $x>1.8$ the polarized gas has lower energy than the unpolarized one, showing that at this point (and higher densities) the ferromagnetic phase is preferred. To the present accuracy we find that both curves cross at $x_{c}^{\prime} \sim 1.8$, which establishes our prediction of the point where the ferromagnetic transition takes place for the hard-sphere Fermi gas. This transition occurs at a density that is close to the estimated freezing density of quantum hard spheres ( $\left.x_{\mathrm{f}} \simeq 1.95\right)$ [34] but slightly below. Our result for $x_{c}^{\prime}$ turns out to be surprisingly close to the original predictions given in Ref. [2], although recent experiments [5] with ${ }^{6} \mathrm{Li}$ atoms seem to indicate that in nature a pairing instability breaks the ultracold nature of the gas before the transition takes place. In any case and as stated above, one should recall that the mechanisms explored here are related but not equal to those found in the experiments which exploit the physical properties of the system close to a resonance.

\section{SUMMARY AND CONCLUSIONS}

To summarize, in this work we have analyzed the possible onset of a phase transition to a ferromagnetic phase for a two-component Fermi gas interacting through a repulsive hard sphere potential. Motivated by recent experimental and theoretical works, we have compared our results with existing predictions [6,13] for a simplified Hamiltonian where only correlations between different spins are allowed. We have shown that, when properly renormalized to correctly account for $p$-wave scattering processes, already existing low-density expansions accurately reproduce Monte Carlo simulations of this simplified model. Based on that, we have shown that $p$-wave terms contribute significantly to the total energy per particle of the polarized and unpolarized systems at $k_{F} a \sim 1$, and that therefore the simplified Hamiltonian for a fully repulsive interaction cannot be used in this range. Finally, we have also presented calculations in the framework of the FHNC equations for a wave function including backflow correlations and an optimized Jastrow factor, showing that a ferromagnetic transition takes place at $k_{F} a \sim 1.8$, surprisingly close to the original prediction of Ref. [2].

\section{ACKNOWLEDGMENTS}

This work has been partially supported by Grants No. FIS2009-07390, No. FIS2008-04403, and No. FIS2008-01661 from MICINN (Spain) and FEDER and Grants No. 2009SGR1003 and No. 2009SGR-1289 from the Generalitat de Catalunya (Spain).
[1] S. Giorgini, L. P. Pitaevskii, and S. Stringari, Rev. Mod. Phys. 80, 1215 (2008).

[2] G.-B. Jo, Y-R. Lee, J-H. Choi, C. A. Christensen, T. H. Kim, J. H. Thywissen, D. E. Pritchard, and W. Ketterle, Science 325, 1521 (2009).

[3] E. Stoner, Philos. Mag. 15, 1018 (1933).

[4] K. Huang, Statistical Mechanics, Sec. 11.7 (Wiley, New York, 1987).

[5] C. Sanner, E. J. Su, W. Huang, A. Keshet, J. Gillen, and W. Ketterle, e-print arXiv:1108.2017v1.

[6] S. Y. Chang, M. Randeria, and N. Trivedi, Proc. Natl. Acad. Sci. (PNAS) 108, 51 (2011).

[7] S. Q. Zhou, D. M. Ceperley, and S. Zhang, Phys. Rev. A 84, 013625 (2011).

[8] T. Sogo and H. Yabu, Phys. Rev. A 66, 043611 (2002).

[9] R. A. Duine and A. H. MacDonald, Phys. Rev. Lett. 95, 230403 (2005).

[10] L. J. LeBlanc, J. H. Thywissen, A. A. Burkov, and A. Paramekanti, Phys. Rev. A 80, 013607 (2009).

[11] G. J. Conduit and B. D. Simons, Phys. Rev. Lett. 103, 200403 (2009).
[12] H. Heiselberg, Phys. Rev. A 83, 053635 (2011).

[13] S. Pilati, G. Bertaina, S. Giorgini, and M. Troyer, Phys. Rev. Lett. 105, 030405 (2010).

[14] X. Cui and H. Zhai, Phys. Rev. A 81, 041602(R) (2010).

[15] C.-C. Chang, S. Zhang, and D. M. Ceperley, Phys. Rev. A 82, 061603(R) (2010).

[16] N. D. Drummond, N. R. Cooper, R. J. Needs, and G. V. Shlyapnikov, Phys. Rev. B 83, 195429 (2011).

[17] G. B. Partridge, K. E. Strecker, R. I. Kamar, M. W. Jack, and R. G. Hulet, Phys. Rev. Lett. 95, 020404 (2005).

[18] S. Rosati, From Nuclei to Particles, Proceedings of the International School of Physics Enrico Fermi, Course LXXIX, Varenna (North Holland, Amsterdam, 1981).

[19] A. Polls and F. Mazzanti, in Introduction to Modern Methods of Quantum Many-Body Theory and Their Applications, edited by A. Fabrocini, S. Fantoni, and E. Krotscheck, Series on Advances in Quantum Many-Body Theory, Vol. 7 (World Scientific, Singapore, 2002).

[20] V. N. Efimov and M. Ya. Amusya, JETP 20, 388 (1965).

[21] A. L. Fetter and J. D. Walecka, Quantum Theory of ManyParticle Systems (McGraw-Hill, New York, 1971). 
[22] R. F. Bishop, Ann. Phys. 77, 106 (1973).

[23] E. Krotscheck, Microscopic Quantum Many-Body Theories and Their Applications, Proceedings of the Valencia School (Springer, New York, 1998).

[24] M. Viviani, E. Buendia, S. Fantoni, and S. Rosati, Phys. Rev. B 38, 4523 (1988).

[25] F. Arias de Saavedra and E. Buendia, Phys. Rev. B 42, 6018 (1990).

[26] E. Manousakis, S. Fantoni, V. R. Pandharipande, and Q. N. Usmani, Phys. Rev. B 28, 3770 (1983).

[27] A. Fabrocini and A. Polls, Phys. Rev. B 25, 4533 (1982).
[28] R. B. Wiringa, V. Fiks, and A. Fabrocini, Phys. Rev. C 38, 1010 (1988).

[29] A. Lovato, O. Benhar, S. Fantoni, A. Yu. Illarionov, and K. E. Schmidt, Phys. Rev. C 83, 054003 (2011).

[30] R. P. Feynman and M. Cohen, Phys. Rev. 102, 1189 (1956).

[31] F. Mazzanti, A. Polls, and A. Fabrocini, Phys. Rev. A 67, 063615 (2003).

[32] R. M. Panoff and J. Carlson, Phys. Rev. Lett. 62, 1130 (1989).

[33] J. Casulleras and J. Boronat, Phys. Rev. Lett. 84, 3121 (2000).

[34] M. H. Kalos, D. Levesque, and L. Verlet, Phys. Rev. A 9, 2178 (1974). 